Reprod. Nutr. Dévelop., 1988, 28 (1), 189-190.

\title{
Absorption du sorbitol par la paroi du rumen chez le mouton
}

Elisabeth RICHET, P. THIVEND, J. LEFAIVRE

Laboratoire de la Digestion des Ruminants, I.N.R.A., Theix, 63122 Ceyrat, France.

Summary. A single dose of 50 or $100 \mathrm{~g}$ of sorbitol was given to four castrated male sheep implanted with chronic catheters in one of the rumen veins; blood sample collection was made during the next hours. Sorbitol levels rapidly increased showing that the polyol was absorbed, but this absorption probably concerned only a slight amount of the dose administered.

Au cours de ces dernières années, le rôle du sorbitol en tant qu'additif alimentaire a été étudié chez les bovins préruminants (Thivend, 1982 ; Thivend et al., 1984), en croissance (Bauchart, Aurousseau et Auclair, 1985 ; Geay, résultats non publiés) ou en lactation (Rémond et Jacquier, 1987). Dans le but d'expliquer ses effets digestifs et métaboliques et son mode d'action, nous avons étudié l'absorption de ce polyalcool au niveau du rumen.

Matériel et méthodes. Quatre moutons mâles castrés (poids vif moyen : $57,5 \mathrm{~kg}$ ) porteurs d'une canule du rumen et d'un cathéter chronique implanté dans une veine du sac dorsal ont reçu directement par la canule $50 \mathrm{~g}$ (3 séries de cinétiques) ou $100 \mathrm{~g}$ (7 séries) de sorbitol au moment de la distribution du repas du matin. La ration constituée de foin de prairie naturelle $(250 \mathrm{~g})$, de luzerne déshydratée $(800 \mathrm{~g})$, de paille de blé $(200 \mathrm{~g})$ et d'orge broyée $(150 \mathrm{~g})$ a été répartie en 2 repas identiques par jour. Vingt-cinq échantillons de sang d'environ $2 \mathrm{ml}$ chacun ont été prélevés au cours des $7 \mathrm{~h}$ suivant le début du repas ; deux cinétiques identiques ont été réalisées après un repas témoin. Toutes ces séries de mesures étaient espacées d'au moins $48 \mathrm{~h}$. Le sorbitol plasmatique a été dosé par méthode spectrophotométrique $(340 \mathrm{~nm})$ en suivant l'apparition de NADH en présence de sorbitol déshydrogénase. Les résultats des cinétiques individuelles sont présentés au tableau 1.

Résultats et discussion. D'une manière générale, la concentration du sorbitol dans le sang de la veine du rumen (en moyenne $15 \mathrm{mg} / \mathrm{l}$ avant le repas) présente, chez les animaux traités, une élévation rapide (maximum atteint en 5 min dans 7 cas sur 10) d'amplitude proportionnelle à la dose de sorbitol ( +19 à $+233 \%$ après $100 \mathrm{~g}$, contre +15 à $+93 \%$ après $50 \mathrm{~g}$ ) malgré une variabilité importante selon les séries de prélèvements (tabl. 1). Le retour relativement rapide (généralement en moins de $30 \mathrm{~min}$ ) des tencurs en sorbitol à des valeurs voisines du taux basal, sans établissement d'un plateau, suggère un catabolisme important de ce polyalcool dans le rumen, puis sous l'action de la sorbitol-déshydrogénase présente au niveau plasmatique et surtout hépatique (Frahm et al., 1977). L'apparition d'une nouvelle élévation modérée et plus tardive des teneurs plasmatiques reflète peut-être une absorption du sorbitol au niveau de la paroi de l'intestin 
grêle. En effet, les concentrations duodénales sont maximales à ce même moment chez des moutons ayant reçu $40 \mathrm{~g}$ en dose unique (Thivend, non publié).

TABL. 1. - Evolution de la concentration plasmatique mesurée dans une veine du rumen après un repas témoin ou additionné d'une dose de sorbitol $(50 \mathrm{ou} 100 \mathrm{~g})$. Les résultats sont exprimés en \% de la valeur observée 5 min avant l'administration $(T-5 ; \mathrm{mg} / \mathrm{ll}$.

\begin{tabular}{|c|c|c|c|c|c|c|c|c|c|c|c|c|}
\hline \multirow{2}{*}{ Temps (min) } & \multicolumn{12}{|c|}{ Dose sorbitol (g) } \\
\hline & \multicolumn{2}{|c|}{0} & \multicolumn{3}{|c|}{50} & \multicolumn{7}{|c|}{100} \\
\hline$T-5$ & 21,7 & 16,3 & 16,7 & 19,6 & 12,1 & 4,2 & 9,2 & 3,8 & 15,8 & 21,7 & 13,9 & 19,2 \\
\hline T 2 & - & - & 122 & 115 & 193 & - & 127 & - & - & - & - & \\
\hline T5 & 87 & 110 & 105 & 109 & 97 & - & 197 & 132 & 119 & 177 & 174 & 202 \\
\hline T 10 & 81 & 97 & 100 & 89 & 145 & - & 142 & - & 84 & 112 & 232 & 158 \\
\hline T 15 & 87 & 90 & 90 & 83 & 138 & 252 & - & 153 & 92 & - & 218 & 132 \\
\hline$T 30$ & 69 & 87 & 75 & 79 & 114 & 333 & 66 & - & 97 & 71 & 145 & 93 \\
\hline T 60 & 71 & 82 & 87 & 105 & 141 & 205 & - & 126 & 90 & 67 & 126 & 87 \\
\hline T 75 & 81 & 85 & 90 & 110 & 141 & 205 & - & - & 90 & 77 & 135 & 80 \\
\hline Т 90 & 81 & 107 & 92 & 109 & 135 & - & - & - & 98 & 83 & 144 & 89 \\
\hline T 120 & 75 & 112 & 100 & 116 & 135 & - & - & 142 & 119 & 81 & 141 & 89 \\
\hline T 150 & 73 & 112 & 100 & 96 & 131 & - & - & - & 119 & 81 & 159 & 91 \\
\hline
\end{tabular}

- : Prélèvements manquants ou hémolysés.

En l'absence d'une part de mesures de concentrations artérielles, et d'autre part de données concernant le débit sanguin dans les veines du rumen, il n'est pas possible de calculer la quantité de sorbitol absorbée par le rumen.

La mise en évidence de l'absorption du sorbitol par la paroi du rumen, échappant ainsi aux dégradations par les microorganismes permet de mieux comprendre la similitude des effets métaboliques de ce polyalcool observés chez les Ruminants et les Monogastriques. II peut ainsi indirectement contribuer à une orientation différente du métabolisme hépatique et/ou tissulaire qui pourrait être à l'origine de I'hypocholestérolémie, l'hypoglycémie et de l'hyperinsulinémie observées à la fois dans ces deux groupes.

Remerciements. - Aux Etablissements Roquette Frères (62136 Lestrem, France) pour la fourniture du sorbitol et pour l'aide financière apportée à la réalisation de ce travail ainsi qu'à $M$. Fabre pour sa collaboration technique.

Bauchart D., Aurousseau B., Auclair E., 1985. Reprod. Nutr. Dévelop., 25, 411-425.

Frahm K., Graf F., Krausslich H., 1977. Zb/. Vet. Med., 24, 81-87.

Rémond B., Jacquier C., 1987. Ann. Rech. Vét., 18, 91-97.

Thivend P., 1982. Bull. Techn. C.R.Z.V. Theix, I.N.R.A., 50, 47-50.

Thivend P., Debarre M., Lefaivre J., Toullec R., 1984. Can. J. anim. Sci., 64 (Suppl.), 102-103. 